\title{
Millimeter-wave channel model simulator for multi-antenna terrestrial links
}

\author{
Dimitrios Ntaikos, Bobby Gizas, George Papageorgiou, Constantinos B. Papadias \\ Athens Information Technology (AIT), \\ Athens, Greece, \\ Email: dint,bogi,gepa,cpap@ait.gr
}

\begin{abstract}
This work introduces a channel model simulator for multi-antenna terrestrial links. The simulator is primarily implemented for the mmWave bands of 19 and $28 \mathrm{GHz}$; however, it could be further employed for other frequency bands as well. The components that are considered include: a) scattering effects due to clusters of scatterers, b) specular and diffused ground reflection, c) oxygen absorption, d) rain attenuation. One of the advantages of this simulator is its flexibility to use simulated as well as actual far field radiation patterns and produce the channel coefficients for various scenarios.
\end{abstract}

\section{INTRODUCTION}

In this paper we will present a channel model simulator that was developed within the H2020 project SANSA [1], in order to simulate multi-antenna channels for terrestrial transmission at millimetre wave frequencies (mmWave) and in particular at $19 \mathrm{GHz}$ and $28 \mathrm{GHz}$. The simulator takes into account several phenomena that occur in a realistic environment at mmWave. At lower frequencies channel models have been extensively studied and various simulators have been already developed. However, at higher frequencies, multi-antenna channel modelling is relatively unexplored.

Due to the directivity of the antenna beams as well as the wavelength at such frequencies, the SANSA channel modelling has prominent differences from those corresponding to lower ones. The most important ones are: a) less scattering effects, b) dominant reflections (ground or other surfaces), and c) differences in the scale of the objects on which the scattering occurs (due to the wavelength). As a result, only a few combined multipaths exist and the use of the central limit theorem cannot be justified. Hence, the received signal, which describes the channel, does not necessarily follow a complex Gaussian distribution and the channel impulse response cannot be assumed Rayleigh distributed. Therefore, conventional distributions are no longer suitable for this model. Since future generation communications are required to operate at higher frequencies, there has been an increased interest and research effort in order to determine the distribution of the channels impulse response $\mathrm{h}_{n}$.

The SANSA 3D channel model simulator addresses all of the above challenges and provides results for the propagation channels at mmWave. The frequencies that have been specifically implemented are the K-band, which corresponds to the terrestrial backhauling and the satellite downlink at $19 \mathrm{GHz}$, and the Ka-band, which is used for the satellite uplink at $28 \mathrm{GHz}$. In particular, the following challenges are addressed:

- Line-of-sight (LOS) propagation

- Ground reflection (flat and rough surfaces)

- Reflections that occur due to the existence of large surfaces and objects, e.g. buildings

- Scattering effects and clusters

- Rain attenuation, oxygen absorption, etc.

\section{Summary OF THE CHANNEL MOdEL SIMULATOR}

The simulator is a channel model application that was designed for use in the context of SANSA and thereafter. Since the research society is still in a premature stage in terms of fully characterizing the numerous frequency bands in the mmWave spectrum, there are limited available databases on channel measurements for frequency bands above 10GHz. This tool can assist in the research by simulating the channel impulse responses for various multi-user scenarios and different topologies.

The model is parameterized using results of extensive sets of measurements. By exploiting any available measurements, it can parameterize frequency-dependent parameters and estimate the propagation mechanism to extract the channel coefficients. Moreover, it can simulate existing infrastructures and environments by placing a number of bidirectional nodes, reflecting surfaces and obstructing objects of different sizes in a 3D coordinated area centered at $(\mathrm{x}, \mathrm{y}, \mathrm{z})$.

It should also be noted that the simulator tool is antenna-independent, in the sense that a channel can be simulated for any type of antenna given its radiation pattern (provided in a separate file). This method introduces simplicity and flexibility and allows us to compare different antennas under the same scattering conditions. With this approach, the user can either select an existing antenna type or design one that matches the needs in a specific environment and conditions. For example, it could be an antenna with a narrower beam or one with a number of nulls at certain directions that could help suppress unwanted interference from neighboring nodes while using the same frequency band. 
Our goal is to build a general interactive tool that exploits, as much as possible, measurements that have been shared by others in similar channel model efforts. Channel modelling is much more complex at mmWave frequencies, compared to other bands positioned in the lower side of the microwave spectrum (10GHz or below). For this reason only a few channel models have been recently introduced. Among the most notable are: METIS 2020 [2], COST2100 / COST [3], IC 1004 [4], NIST 5G [5], MiWEBA [6], ETSI mmWave SIG [7], mmMagic [8], NYC Wireless [9], Quadriga [10].

The SANSA simulator targets at the $19 \mathrm{GHz}$ and $28 \mathrm{GHz}$ frequency bands. The user-defined variables that are considered in the model (for both frequencies) are:

- Number of $\mathrm{T}_{x} / \mathrm{R}_{x}$ users to simulate (multi-user)

- Applicable scenario to simulate (urban, suburban, rural) and number of clusters, $\mathrm{n}_{c}$

- The location (coordinates $(\mathrm{x}, \mathrm{y}, \mathrm{z}))$ of the users, base stations and clusters in the 3D environment

- Rain rate $(\mathrm{mm} / \mathrm{hr})$

- Oxygen absorption factor $(\mathrm{dB} / \mathrm{km})$.

Parameters such as number of clusters, intra-cluster subpath count and rain loss coefficients, which are related to the selected frequency, have been considered according to the work reported in [4], [6], [11] and [12]. However, the simulator could be further utilized so that it models environmental characteristics of other mmWave bands as well, with the use of lookup tables that correspond to the respective frequency.

\section{GRAPHICAL USER INTERFACE}

In order to provide a more user friendly tool we have implemented the SANSA simulator graphical user interface (GUI), which is a compiled standalone application that was build using MATLAB code. It can be installed in any Windows-based computer without requiring any licensing or software installation other than the application itself. A snapshot of the GUI is demonstrated in Figure 1.

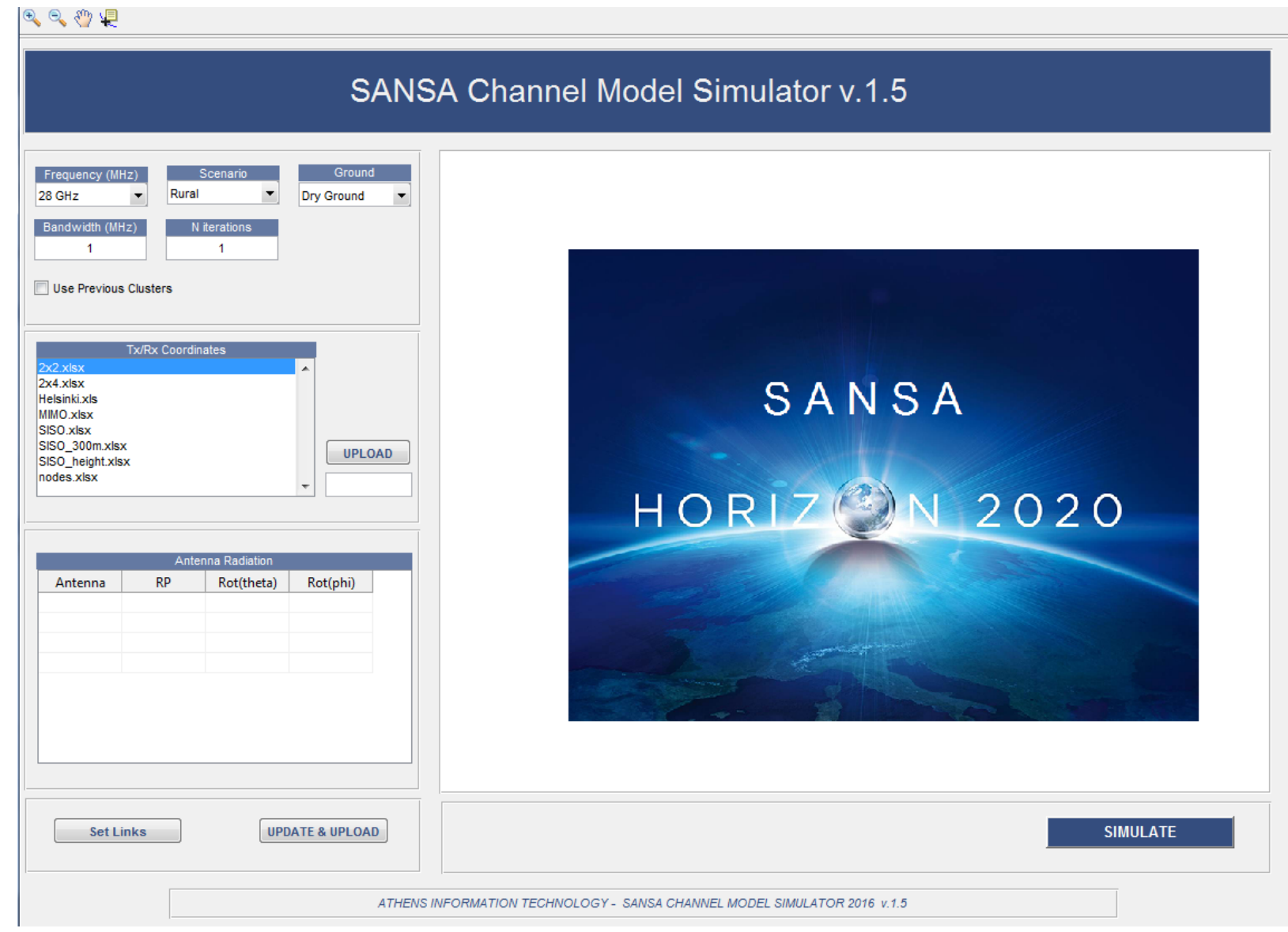

Fig. 1: GUI of the SANSA channel model simulator. 
TABLE I: Node coordinates for a $2 \times 4$ network topology

\begin{tabular}{l|c|c|c|c}
\hline Node & Type & $\mathrm{X}(\mathrm{m})$ & $\mathrm{Y}(\mathrm{m})$ & $\mathrm{Z}(\mathrm{m})$ \\
\hline \hline 1 & $\mathrm{~T}_{x}$ & 500 & 500 & 4 \\
2 & $\mathrm{~T}_{x}$ & 500 & 1000 & 4 \\
\hline 3 & $\mathrm{R}_{x}$ & 700 & 400 & 4 \\
4 & $\mathrm{R}_{x}$ & 700 & 600 & 4 \\
5 & $\mathrm{R}_{x}$ & 700 & 1100 & 4 \\
6 & $\mathrm{R}_{x}$ & 700 & 1200 & 4 \\
\hline
\end{tabular}

\section{Rx Antennas}

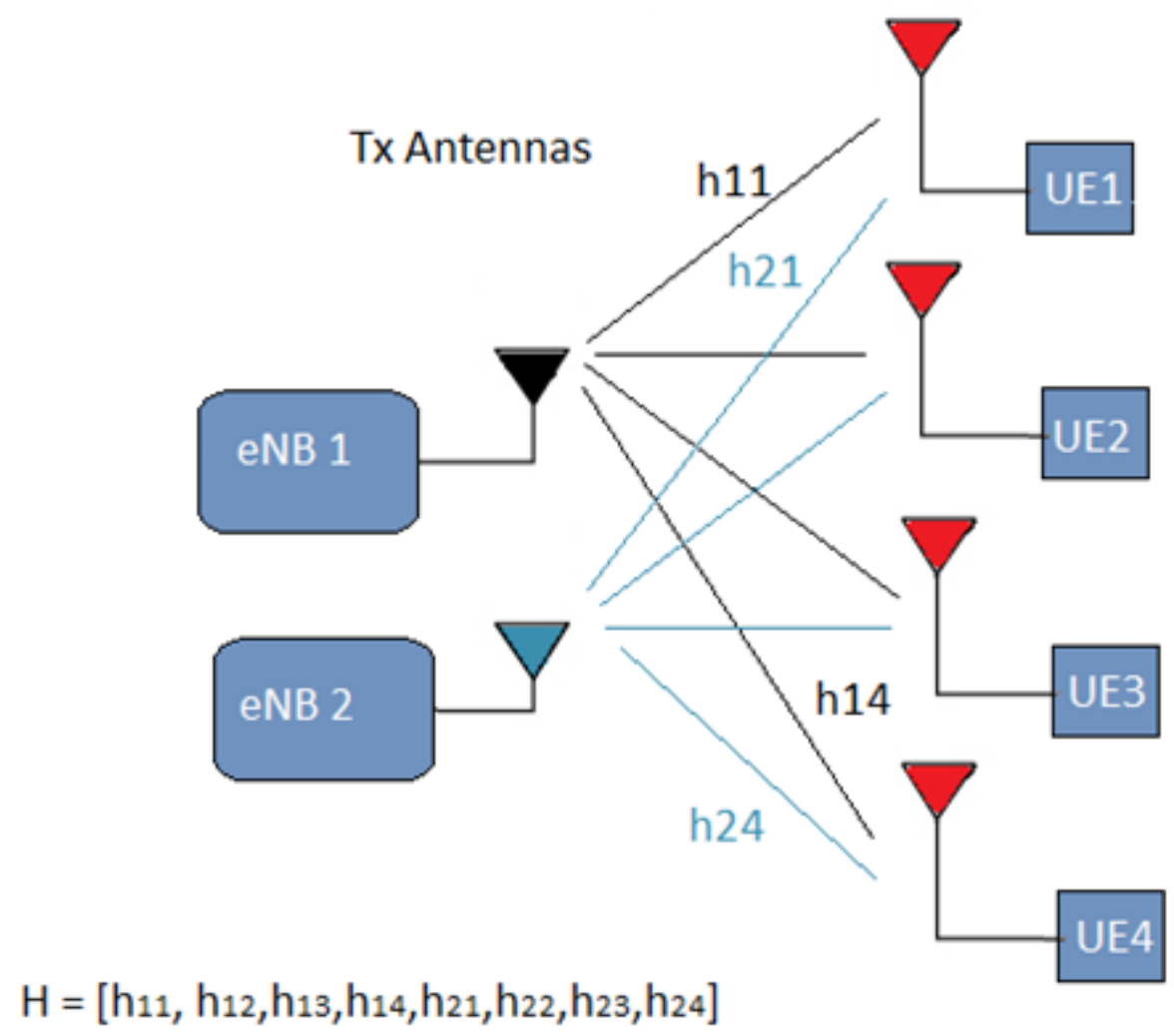

Fig. 2: Example of a $2 \mathrm{~T}_{x} / 4 \mathrm{R}_{x}$ network with its associated channels.

\section{INPUT PARAMETERS}

The simulator's requirement for the scattering environment to be configured for any given topology is met by defining a number of parameters in advance (see Table I). Initially, a file that includes the node locations must be edited, saved and uploaded. Next, a list of configuration settings, which includes parameters such as the node type, antenna radiation patterns and the azimuthal and elevation angles, is updated. After the completion of this step each node is configured properly and the simulator acquires knowledge of the number of transmitters and receivers to be used. Finally, the tool simulates all possible combination of $\mathrm{R}_{x} / \mathrm{T}_{x}$ nodes and produces the channel impulse responses that are saved to a file in the form of channel coefficients $\left(h_{11}, h_{21}, h_{31}, \ldots\right)$. In Table $I$, the tool simulates a total of eight channel impulse responses (CIRs), since there are two transmitter and four receiver nodes in total (see Figure 2). Figure 3 illustrates the used radiation pattern plots for the eight nodes of the $2 \mathrm{~T}_{x} / 4 \mathrm{R}_{x}$ of the network in Figure 2.

Other parameters that should be predefined are: a) the type of scenario (rural, urban, etc.), b) the transmit/received power, c) the simulation bandwidth and d) the rain rate. The selection of the scenario serves the purpose of defining the number of clusters that match any given environment, such as a city center, a low-rise residential neighborhood or a remote countryside 


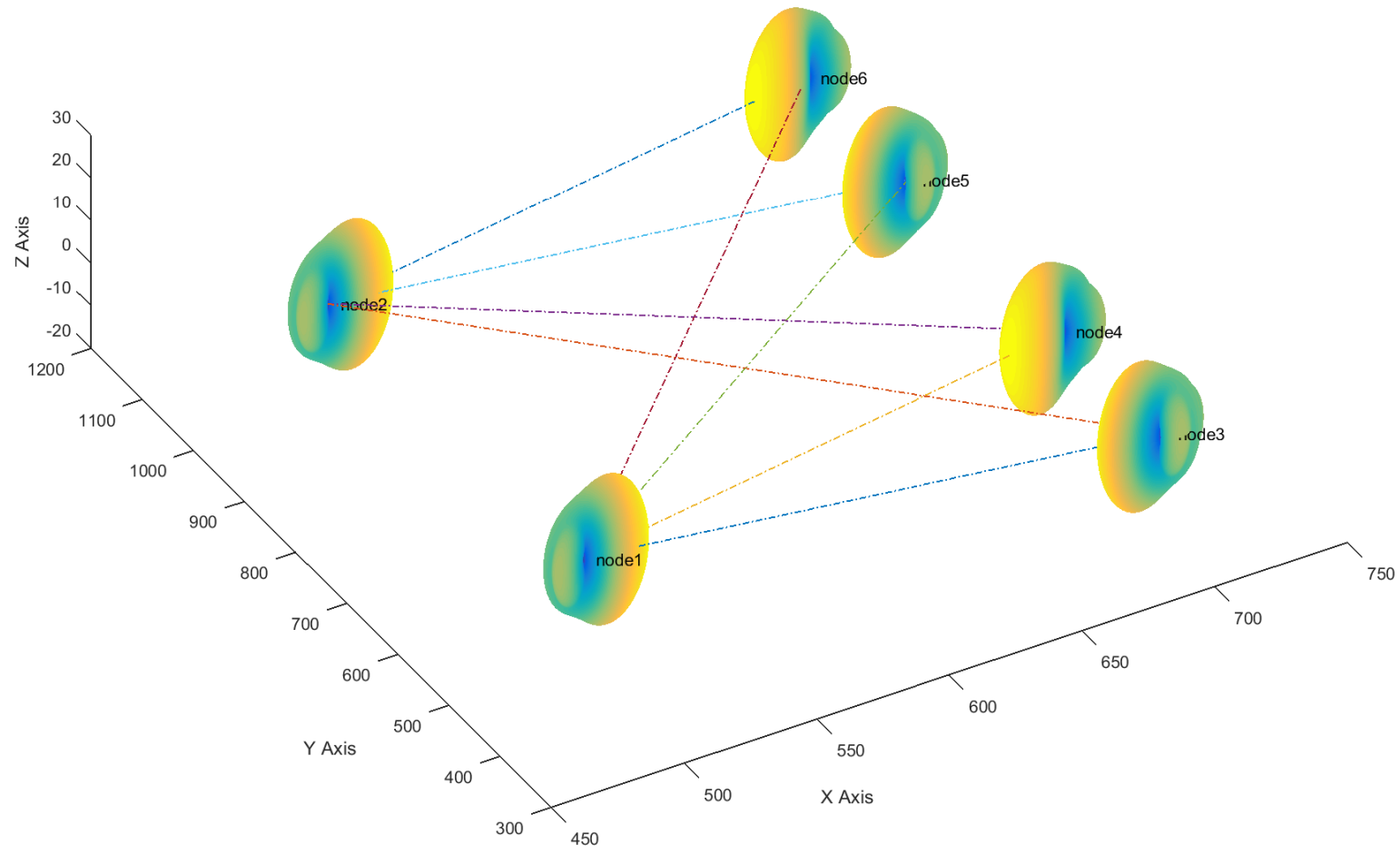

Fig. 3: Example of a $2 \mathrm{~T}_{x} / 4 \mathrm{R}_{x}$ network plot (see the coordinates in Table I).

location. The transmit power and rain rate can be adjusted to any level, while the bandwidth can be set to any value under the assumption that all frequency-dependent parameters (such as cluster properties, losses, etc.) do not deviate significantly from the center frequency.

The final step is the selection of a radiation pattern from the list of available antennas, for each node; it also includes the configuration of their azimuthal and elevation angles.

\section{Modelling THE SCATtering ENVIRONMENT}

Once the parameters have been configured and saved the simulator uses the coordinates of the nodes to distribute a number of clusters that emulate objects that reflect or obstruct the propagating wave (scattering).

At mmWave frequencies, the specular reflection component that occurs in lower frequency remains; however, more pronounced diffuse components will appear as well. The law of reflection accounts for the specular reflection component and the diffuse reflected components appear from the radiation impinging on surface imperfections on the order of the wavelength of propagation, read [11]. Once the cluster coordinates are randomly selected the simulator distributes, within a few meters, more scattering points that will emulate the multipath components / diffused subrays, as demonstrated in Figure 4.

At the final step, the simulator calculates the distances and angles between the scatterers and each transmitter and receiver node. For each multipath component that arrives the phase angle is computated based on the travelled distance, while the reflected signal's amplitude is chosen to be Rayleigh distributed, read [6].

\section{Vi. Time Clusters and Multipath Properties}

As already discussed, the channel model is clustered-based. The number of clusters is heavily dependent on the type of simulated scenario and the frequency. Time clusters are formed by a group of rays travelling closely in time over all directions. The number of time clusters corresponds to the number of propagation paths that the electromagnetic energy follows to travel 


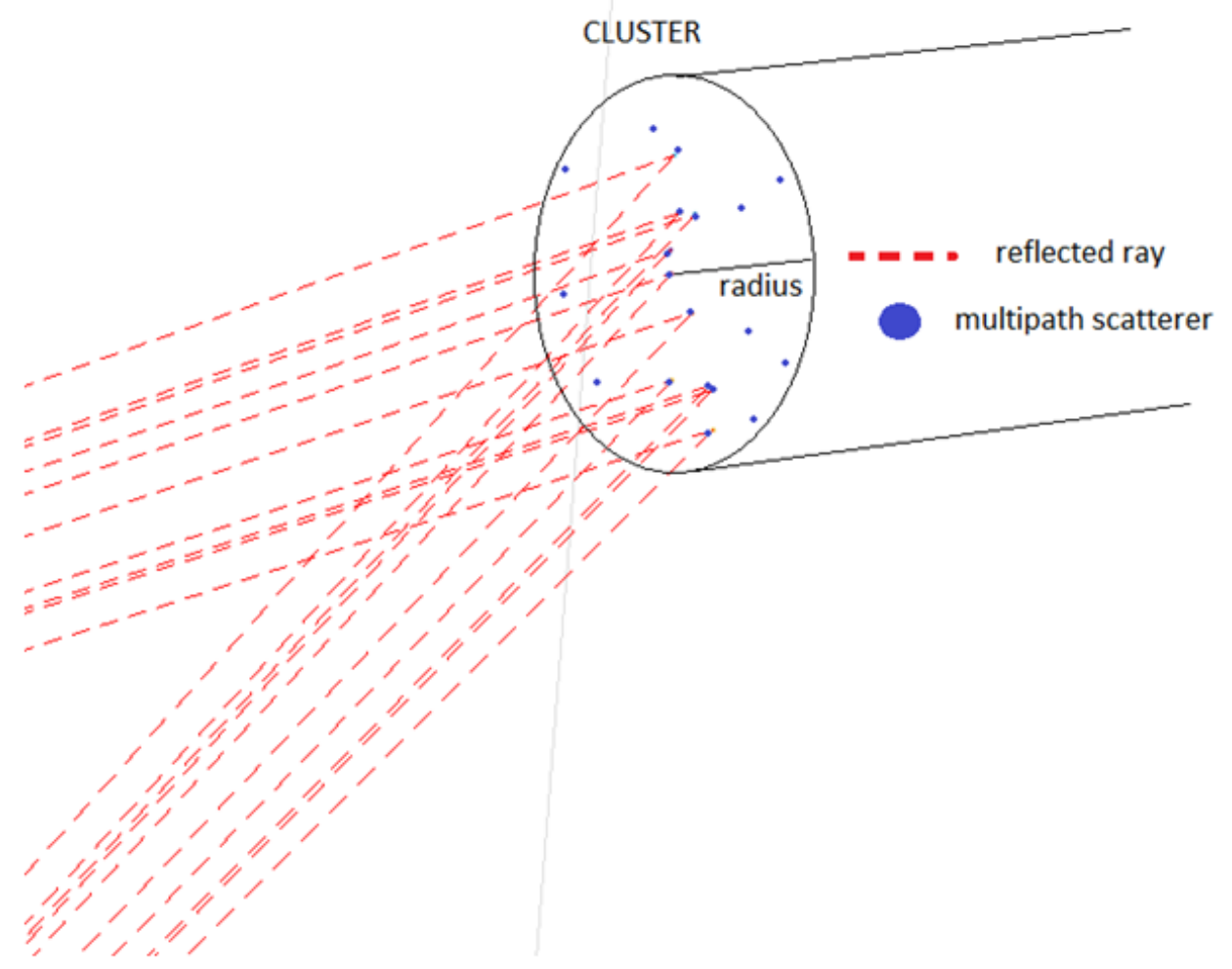

Fig. 4: Multipath components of a single cluster.

from the transmitter, through the clusters, to the receiver. They are composed of several multipath components, which arise from electromagnetic reflections upon obstructions which typically exhibit macroscopic roughness [13], and upon impact of such obstructions, the radiation will tend to break up into microscopic subpath components. The number of subpaths at each time cluster corresponds to the number of subpath components contained within one time cluster.

For any given scenario such as the urban, the suburban or the rural environment, the simulator will place a certain number of clusters, accordingly. Each cluster, corresponding to an object in the real world (e.g. buildings, trees etc.) is modelled by many smaller scatterers. In that sense, multiple rays arrive and depart at each cluster.

\section{Channel Model Path Loss Analysis}

The three main components that characterize the propagation of a signal in free space are: a) the line-of-sight (LOS), which is the most dominant (travels the least distance), b) the ground reflection that could be modelled as a specular ray or a combination of the specular and the diffused rays (depending on the surface's roughness) and c) a cluster of multipath components (reflections by a large object), read [14].

For the estimation of the channel impulse response the simulator calculates the LOS, the ground reflection and all the multipath component properties. These properties include the distance, the angle of arrival (AoA), the angle of departure (AoD), the phase and the delay, read [15]. The distance corresponds to the path length that the signal travels from the transmitter to the scatterer (where it is reflected or partially absorbed) and from the scatterer to the receiving end. Taking into account the total distance, it computes the path loss and includes losses encountered due to scatterer absorption, rain attenuation, oxygen absorption, etc.

Considering all types of the aforementioned effects the total path loss equation (in $\mathrm{dB}$ ) is given by [16]:

$$
P L=P_{T_{x}}-P_{R_{x}}+G_{T_{x}}+G_{R_{x}}+20 \log _{10} \frac{\lambda}{4 \pi D}+S_{a b s}+A_{a t t},
$$

where $P$ denotes the power, $G$ the antenna gains (indexed by $\mathrm{T}_{x}$ and $\mathrm{R}_{x}$ for transmitter and receiver, respectively), $S_{a b s}$ the scatterer absorption factor, $A_{a t t}$ the rain attenuation, $\lambda$ the wavelength and $D$ is the distance. 


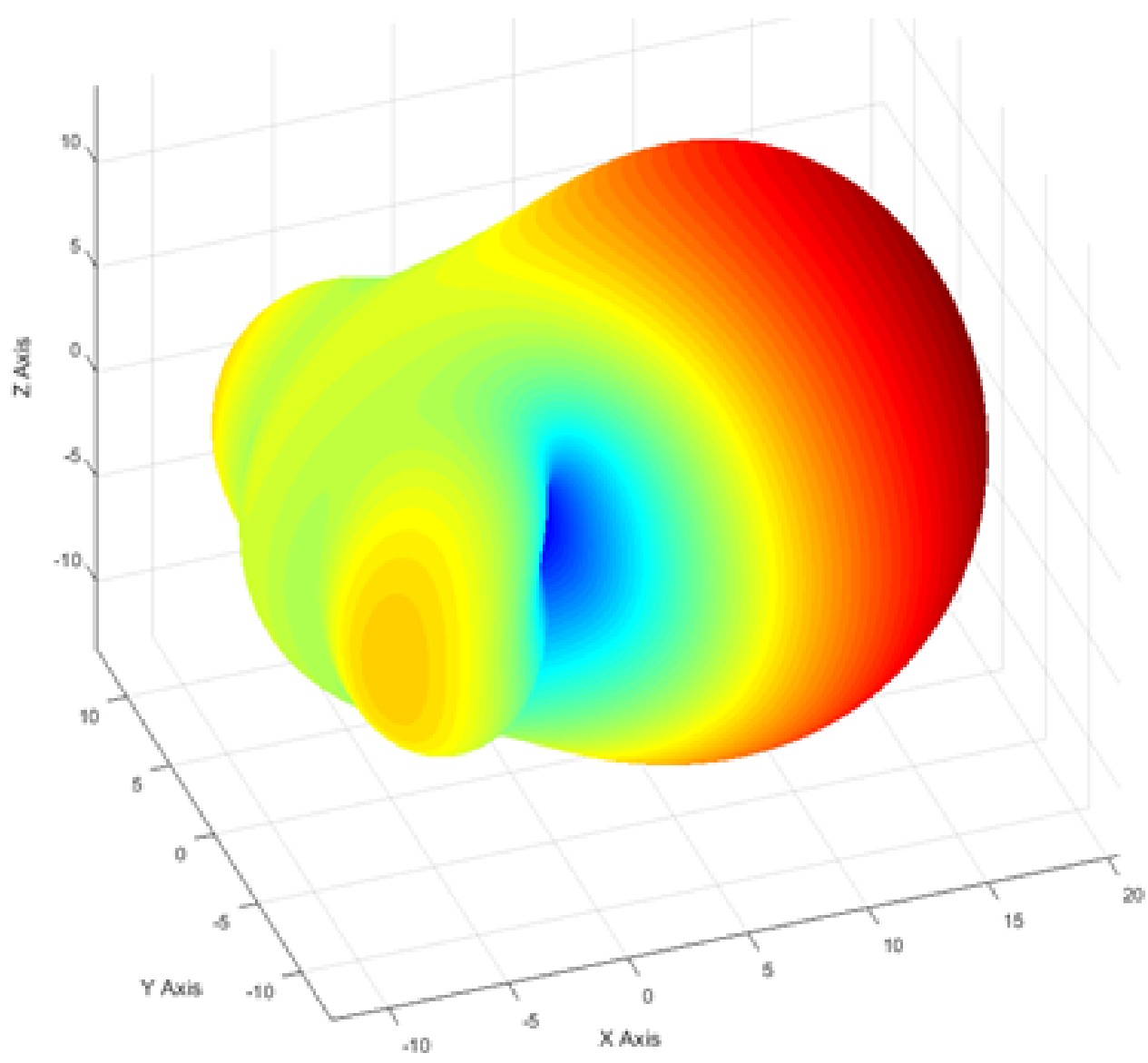

Fig. 5: Radiation pattern of a bowtie antenna at $19 \mathrm{GHz}$.

The departure and arrival angles are introduced as a function of azimuthal $(\theta)$ and elevation $(\phi)$ angles. These angles are calculated via the following mathematical expressions:

$$
\begin{gathered}
\theta_{T_{x}}=\tan ^{-1} \frac{P_{2}(y)-P_{1}(y)}{P_{2}(x)-P_{1}(x)}, \phi_{T_{x}}=\tan ^{-1} \frac{P_{2}(z)-P_{1}(z)}{P_{2}(x)-P_{1}(x)} \\
\theta_{R_{x}}=\tan ^{-1} \frac{P_{2}(y)-P_{3}(y)}{P_{2}(x)-P_{3}(x)}, \phi_{R_{x}}=\tan ^{-1} \frac{P_{2}(z)-P_{3}(z)}{P_{2}(x)-P_{3}(x)}
\end{gathered}
$$

The phases and delays for each received multipath, including the LOS and ground reflections, are calculated via the following formulas:

$$
d_{n}=\frac{2 \pi c}{f}\left(D-D_{L O S}\right), \text { and } \tau_{n}=\left(\frac{D f}{\lambda}\right),
$$

respectively, where $c$ is the speed of light, $D$ is the distance and $f$ is the center of the frequency band.

As already discussed, the channel model simulator is antenna independent. It makes use of the radiation pattern generated by any type of antenna with an arbitrary geometry and characteristics, as well as theoretical radiation patterns. An antenna radiation pattern is loaded through a file in the form of $(\theta, \phi$, gain). After the calculation of the AoA and AoD for each multipath component, the simulator extracts the effective individual antenna gains and calculates the path loss. This is accomplished by extracting the corresponding value of the gain at a particular combination of antenna's azimuthal and elevation angles. An example of the far-field radiation pattern at $19 \mathrm{GHz}$ for an antenna array is shown in Figure 5.

An additional effect that is also taken into account is the time dispersion caused by the multipath channels. Each copy arrives at the receiver with a different amplitude and goes through a different phase shift, which produces a different excess delay. The power delay profile is the channel power spectrum density, which is a function of delay. In Figure 6 , we present three distinct time clusters with maximum intra-cluster delay of $20 \mathrm{nsec}$ for a suburban environment. 


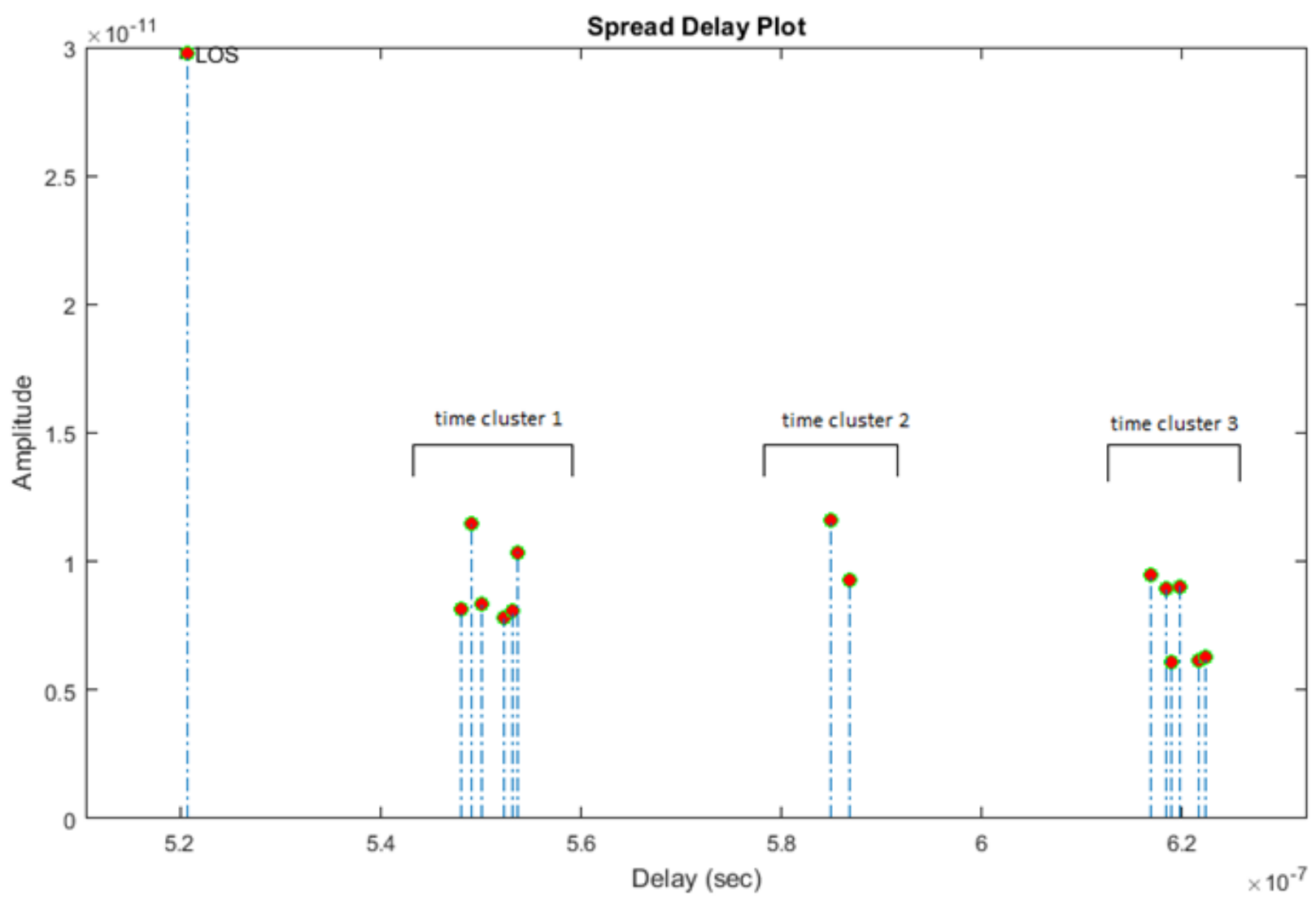

Fig. 6: Spread delay simulation results for three time clusters.

Finally, the simulator estimates the channel impulse response and saves the channel coefficients in a separate file. The channel impulse response model can be analytically expressed as:

$$
h(t, \tau)=\sum_{n=1}^{N} a_{n}(t, \tau) e^{j 2 \pi f_{c} \tau_{n}(t)-\frac{j 2 \pi d_{n}}{\lambda}} \delta\left(\tau-\tau_{n}(t)\right),
$$

where $N$ is the number of multipath components, $a_{n}$ is the attenuation due to path loss and $\tau_{n}=d_{n} / c$ is the delay with respect to each scatterer or reflector. The channel response is modelled as the summation of all the arriving multipath components.

\section{ViII. Rain AtTEnuAtion}

Rain fade is one of the most significant and adverse propagation effects that degrades the performance of a wireless communication system that operates at mmWave frequencies. In order to efficiently plan and utilize the wireless link, the rain attenuation must be studied and included in the link budget. As the frequency of operation increases the wavelength of the travelling signal decreases, therefore it becomes significant comparable to rain droplet size and their separation distances. This phenomenon has a greater effect on frequencies above $10 \mathrm{GHz}$ where the signal strength is highly attenuated, especially in heavily downpour conditions. The attenuation encountered by the factor of rain can be estimated by:

$$
A_{\text {rain }}=\gamma_{R} D_{\text {rain }}(d B)
$$

where $\gamma_{R}$ is know as the specific attenuation factor and $D_{\text {rain }}$ is the link's path distance. The specific attenuation factor is given by:

$$
\gamma_{R}=k R^{a}(d B / k m)
$$

where $k, a$ are frequency depended coefficients and $R$ is the rain rate measured in $\mathrm{mm} / \mathrm{hr}$, as shown in Table II. Due to the non-spherical nature of rain droplets the attenuation is much higher in horizontally polarized waves compared to the vertical ones. 
TABLE II: Urban environment scenario: the road tunnel model

\begin{tabular}{l|c|c|c|c}
\hline & \multicolumn{2}{|c|}{ Horizontal Polarization } & \multicolumn{2}{c}{ Vertical Polarization } \\
\hline \hline Freq $(\mathrm{GHz})$ & $k$ & $a$ & $k$ & $a$ \\
\hline 10 & 0.0101 & 1.2760 & 0.0089 & 1.2640 \\
20 & 0.0751 & 1.0990 & 0.0601 & 1.0650 \\
28 & 0.1870 & 1.021 & 0.1670 & 1.0000 \\
40 & 0.3500 & 0.9390 & 0.3100 & 0.9290 \\
\hline
\end{tabular}

TABLE III: Simulation Parameters

\begin{tabular}{l|l}
\hline $\mathrm{T}_{x}-\mathrm{R}_{x}$ Distance $(\mathrm{m})$ & 300 \\
\hline Number of Clusters & 5 (Urban Scenario) \\
\hline Height $\mathrm{T}_{x}(\mathrm{~m})$ & 5 \\
\hline Height $\mathrm{R}_{x}$ & 3.5 to 5 (steps of $0.5 \mathrm{~m})$ \\
\hline Ground Type & Dry asphalt \\
\hline Antenna $\mathrm{T}_{x} / \mathrm{R}_{x}$ & Isotropic/Isotropic \\
\hline
\end{tabular}

\section{OXYGEN ABSORPTION}

The specific attenuation at frequencies up to $1000 \mathrm{GHz}$, due to dry air and water vapor, can be evaluated most accurately at any value of pressure, temperature and humidity. This evaluation is accomplished by means of a summation of the individual resonance lines from oxygen and water vapor. Small additional factors for the non-resonant Debye spectrum of oxygen below $10 \mathrm{GHz}$, pressure-induced nitrogen attenuation above $100 \mathrm{GHz}$ and a wet continuum to account for the excess water vapor absorption found experimentally, are also taken into account. At the millimeter wave frequency of $28 \mathrm{GHz}$, the absorption is very small for short distances (around $0.2 \mathrm{~dB} / \mathrm{km}$ ). At higher frequencies $(60 \mathrm{GHz})$ thought, the oxygen can attenuate signals by more than $15-20 \mathrm{~dB} / \mathrm{km}$, although millimeter wave systems are usually planned for much smaller distances, see [12].

\section{Measurements And Results}

The signal propagation model is calculated, based on the geometry of the deployment and the location of the nodes, in a deterministic manner with the use of theoretical formulas. Some parameters, such as the reflection, the absorption losses and the total number of cluster subpaths are selected according to a Rayleigh and Uniform distribution, respectively, while the total number of clusters are dependent on the considered scenario. For example, the received power in a rural scenario is calculated as the superposition of the signals received by the LOS and ground reflected paths with the rest of the multipath rays produced by a single cluster. An example for an urban simulation is presented in Table III, where the number of clusters is set to five.

In this example, we generate a topology that includes the locations of each of the bi-directional nodes. The example topology simulates the path loss model of a single point-to-point link with a separation distance of 300 meters. The 2D scattering environment topology for the single point-to-point urban link is demonstrated in Figure 7. In Figure 8 we present the channel frequency response for an $800 \mathrm{MHz}$ bandwidth and in Figure 9 the channel transfer function for various receiver antenna heights.

\section{Channel Simulation on the Helsinki Topology}

In the context of the SANSA project we have also considered the actual Helsinki topology, in order to produce results that could also be verified by real measurements in the future. The simulated results target at the study of the backhaul network architecture. The selected topology is depicted in Figure 10. The Helsinki topology consists of a number of interconnected start topologies; based on the actual data of the database it is composed of 28 links and 15 actual locations (nodes). In order to derive the SINR for each receiver based on the coordinated frequency plan, we employ the SANSA channel model simulator. Using the channel simulator, one could estimate the number of nulls that each SANSA smart antenna must produce in order to tackle the strong interferers due to node co-existence and frequency reuse. An interferer is considered to be harmful, if the level of received interference increases the noise floor by $10 \%$ or more.

The first step for the simulation of the Helsinki topology, is the translation of the longitude and latitude coordinates into X, $\mathrm{Y}, \mathrm{Z}$ values (meters). This is done by selecting a node, which serves as a reference point and by calculating the distance, the azimuthal and elevation angles for all of the existing nodes. The next step requires setting the links between the nodes, based on the connectivity matrix information, and adjusting the antenna beams to point to the correct azimuthal and elevation angles. When all setting are configured correctly, the simulation is performed and exports the channel coefficients that correspond to each $\mathrm{T}_{x}-\mathrm{R}_{x}$ link. The produced data can serve several purposes, e.g., providing the SINR levels of any given topology. An example of the SINR for a point-to-point link between nodes 1 and 2 is shown in Figure 11. As it can be seen, links 2, 4, 5, 9 and 10, desensitize the receiver, since the unwanted signal received by node 1 is above the noise floor. Hence, the smart antenna should be capable of producing 5 beam nulls that can suppress the extra noise caused by the adjacent interfering nodes. 


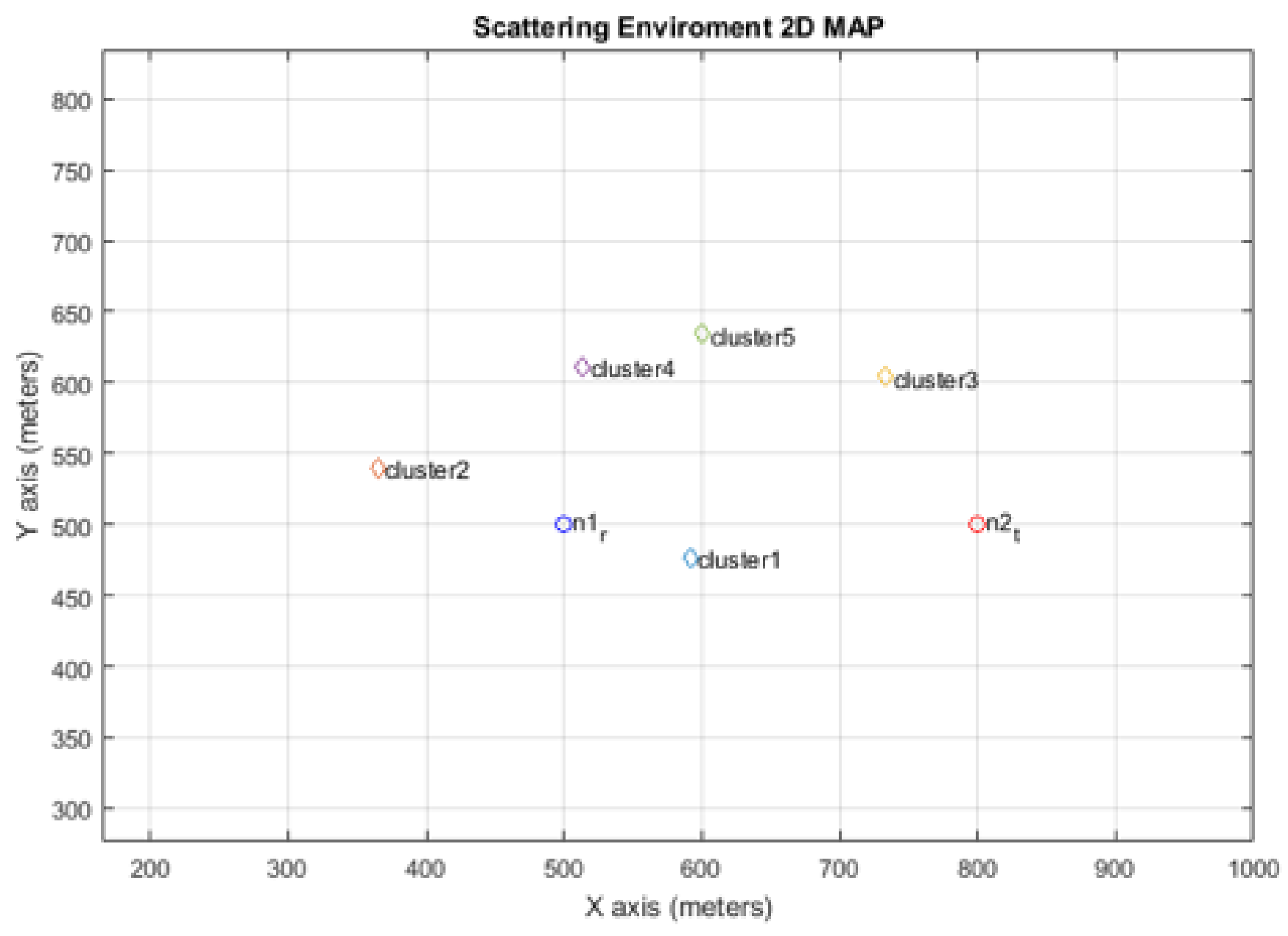

Fig. 7: A 2D scattering environment topology.

\section{Conclusions}

A novel 3D channel model simulator for terrestrial links, in the context of SANSA project, is proposed. We have presented the advantages of the mmWave simulator and we have also demonstrated an application usage on the Helsinki topology. Future directions include: a) the implementation of the Doppler effect for a moving node scenario and b) the consideration of the polarization of the antennas both at the transmitter and the receiver side.

\section{ACKNOWLEDGMENT}

This work was supported by the European Union's Horizon 2020 research and innovation program in the framework of Project SANSA, Grant No 645047.

\section{REFERENCES}

[1] [Online]. Available: http://sansa-h2020.eu/

[2] [Online]. Available: https://www.metis2020.com/documents/simulations/

[3] L. Liu, C. Oestges, J. Poutanen, K. Haneda, P. Vainikainen, F. Quitin, F. Tufvesson, and P. D. Doncker, "The cost 2100 mimo channel model," IEEE Wireless Communications, vol. 19, no. 6, pp. 92-99, December 2012.

[4] [Online]. Available: http://www.ic1004.org/uploads/ ChannelMeasurementsModelingFor5GAbove6GHz.pdf

[5] [Online]. Available: https://www.nist.gov/ctl/5g-mmwave-channel-model-alliance

[6] [Online]. Available: http://www.miweba.eu/

[7] [Online]. Available: http://www.etsi.org/

[8] [Online]. Available: https://5g-mmmagic.eu/

[9] [Online]. Available: http://wireless.engineering.nyu.edu/

[10] [Online]. Available: http://quadriga-channel-model.de/

[11] T. S. Rappaport, G. R. MacCartney, M. K. Samimi, and S. Sun, "Wideband millimeter-wave propagation measurements and channel models for future wireless communication system design," IEEE Transactions on Communications, vol. 63, no. 9, pp. 3029-3056, Sept 2015.

[12] M. Hall, Effects of the troposphere on radio communication, ser. IEE Electromagnetic Waves Series. P. Peregrinus on behalf of the Institution of Electrical Engineers, 1979. 

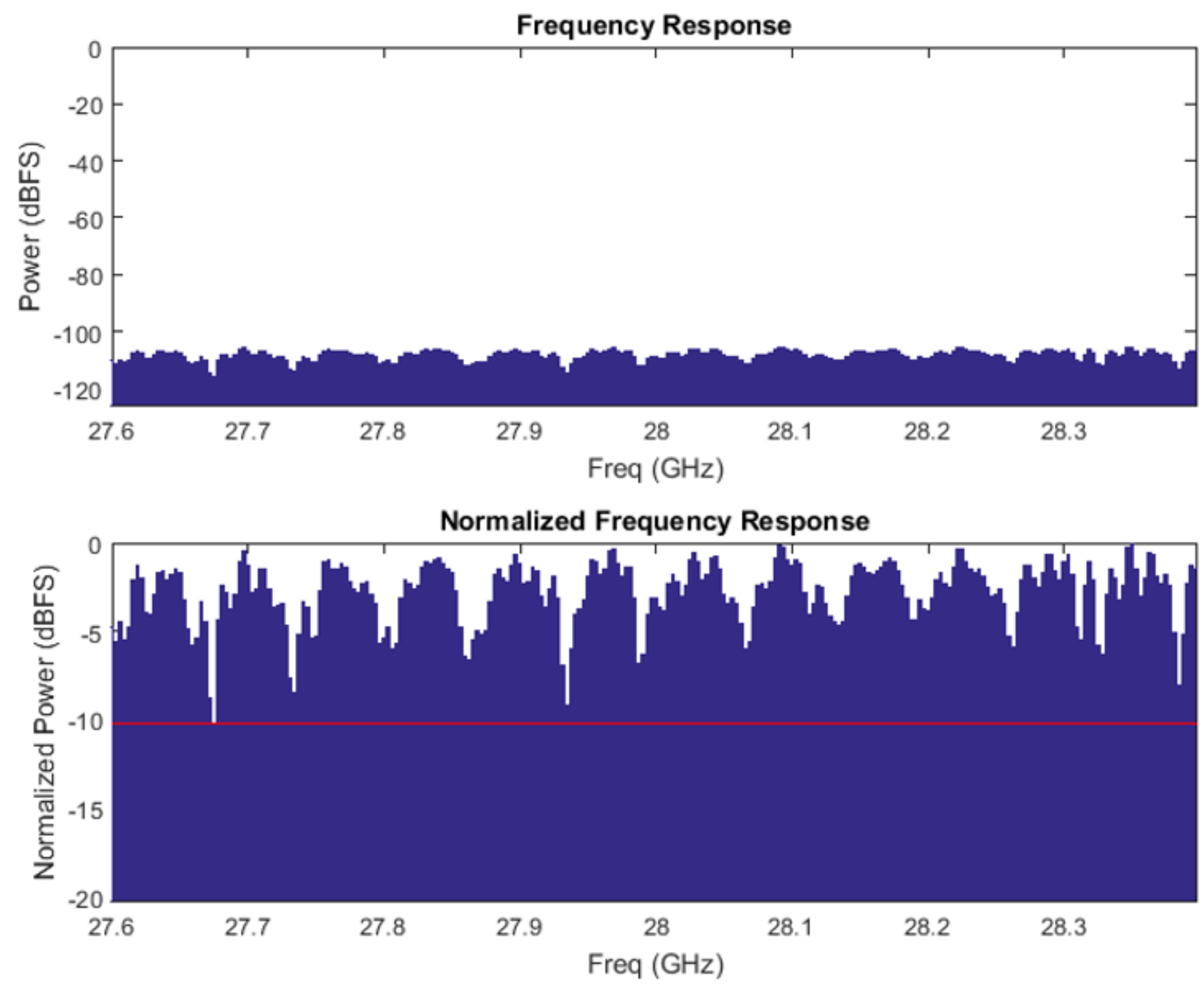

Fig. 8: Channel frequency responce (800MHz bandwidth).

[13] T. S. Rappaport, S. Sun, R. Mayzus, H. Zhao, Y. Azar, K. Wang, G. N. Wong, J. K. Schulz, M. Samimi, and F. Gutierrez, "Millimeter wave mobile communications for 5g cellular: It will work!" IEEE Access, vol. 1, pp. 335-349, 2013.

[14] P. Beckmann and A. Spizzichino, The scattering of electromagnetic waves from rough surfaces. Norwood, MA : Artech House, 1987, reprint. Originally published: Oxford [Oxfordshire] ; New York : Pergamon Press, 1963. (International Series of Monographs on Electromagnetic Waves ; v. 4).

[15] [Online]. Available: https://www.itu.int/rec/R-REC-P.837-6-201202-I/en

[16] A. Goldsmith, Wireless Communications. Cambridge University Press, 2005. 


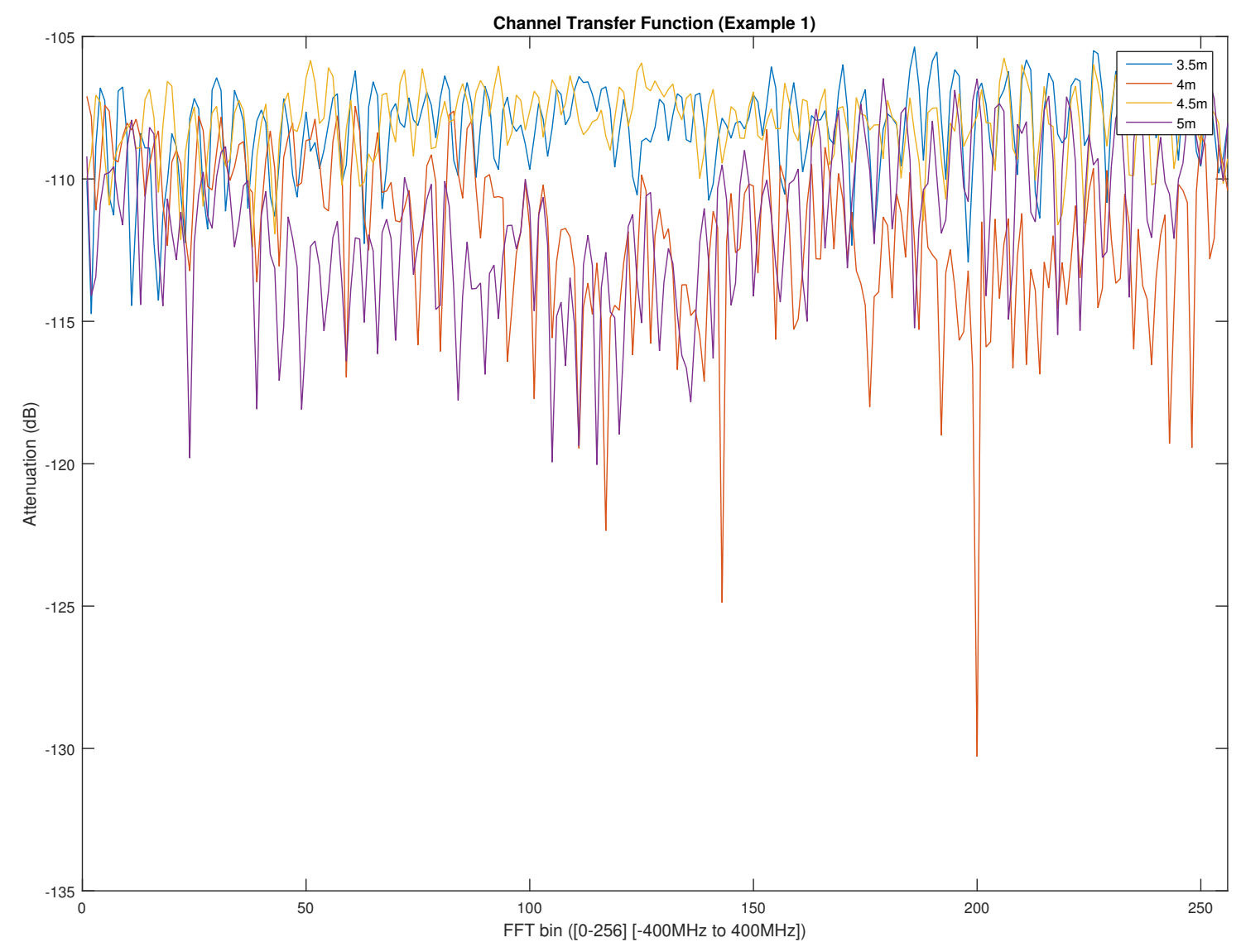

Fig. 9: Channel transfer function (for various receiver heights). 


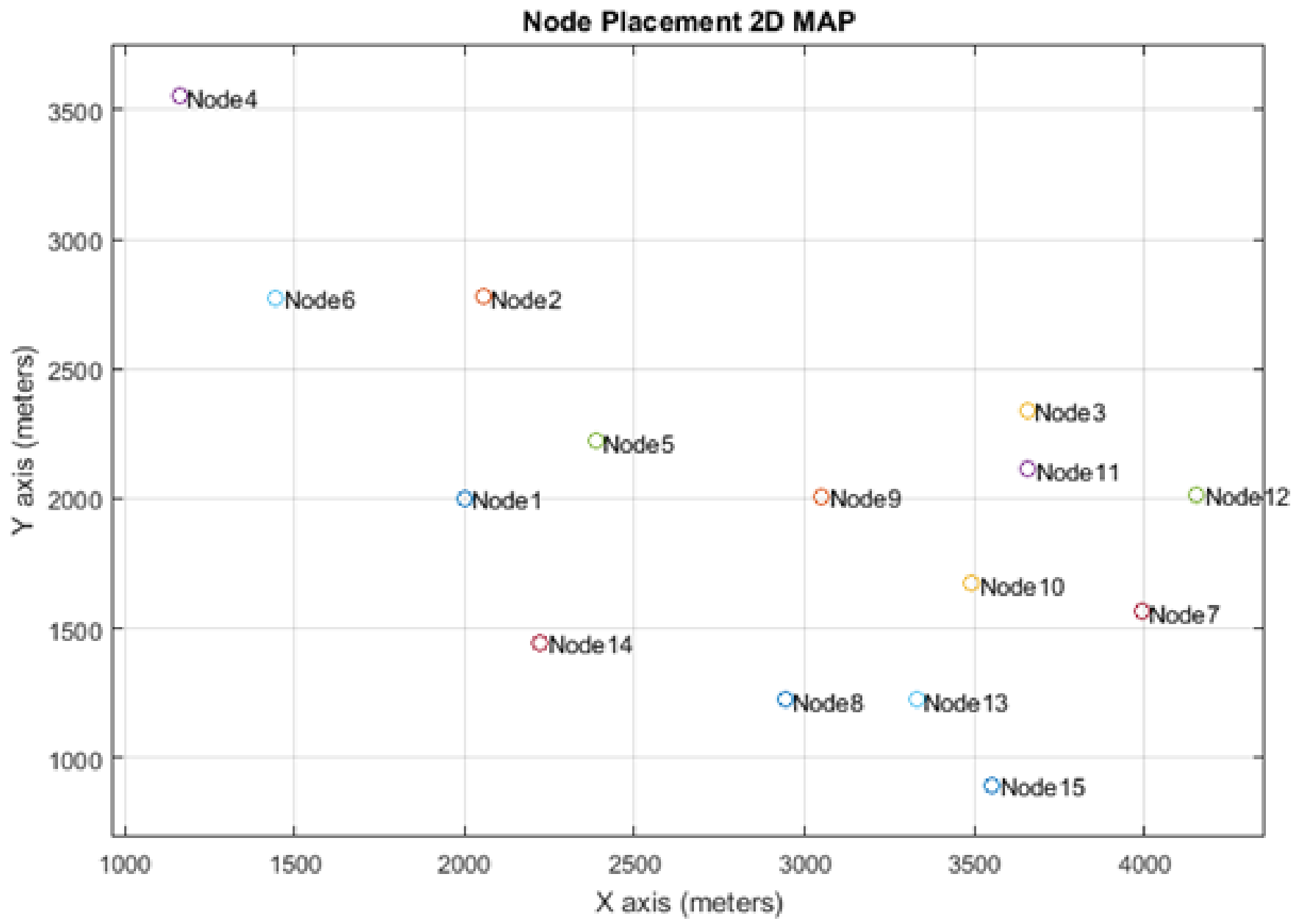

Fig. 10: Helsinki topology. 


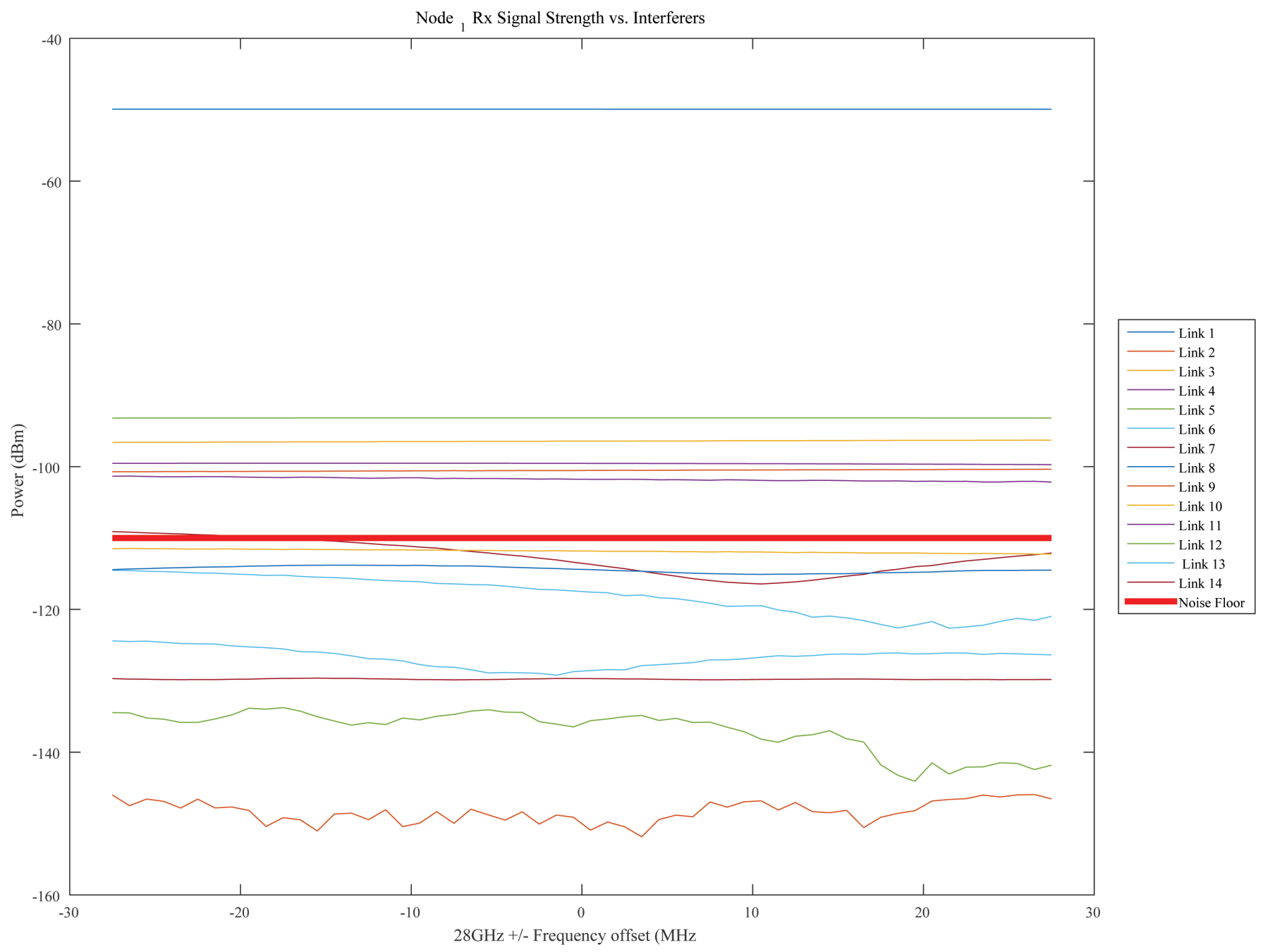

Fig. 11: Node 1, signal strength versus unwanted interferers. 